\title{
La pedagogía de casos como estrategia catalizadora del cambio hacía la autonomía del alumno y del profesor en la formación inicial del profesorado de secundaria
}

\author{
A pedagogia de casos como estratégia \\ catalizadora da mudança para a autonomia \\ do aluno e do professor na formação inicial \\ de professores do ensino secundário
}

\section{Case pedagogy as a catalyst for change towards teacher and learner autonomy in initial teacher training on secondary education}

Manuel Jiménez Raya ${ }^{1}$

\begin{abstract}
RESUMEN
La formación inicial para la profesión docente no solamente implica preparar para conocer, pensar y entender, sino que la preparación para la profesión docente implica ayudar a entender para actuar. Diversos especialistas han recomendado la pedagogía de casos en la formación para la profesión docente inicial, pues puede ayudar a superar algunas de las carencias de los modelos tradicionales de formación y contribuir de manera muy significativa al desarrollo profesional de los profesores. La pedagogía de casos se basa en la idea de que el conocimiento se construye sobre la base del conocimiento previo, va asociado a la experiencia, es transformable y puede cambiar, por lo que ayuda al profesor en formación a entender la complejidad de la docencia, animando a la búsqueda de soluciones docentes alternativas. En
\end{abstract}

DOI: $10.1590 / 0104-4060.49138$

1 Universidad de Granada. Departamento de Filologías Inglesa y Alemana. Granada, España. Facultad de Filosofía y Letras. Campus Cartuja, 18071.E-mail: mjraya@ugr.es 
este trabajo mantengo que la pedagogía de casos es útil para promover la reflexión crítica, facilitar la comprensión de la teoría, y para formar profesores con capacidad de resolución de problemas, favoreciendo la conexión entre enseñanza, investigación y desarrollo profesional.

Palabras-clave: formación de profesores; pedagogía de casos; formación inicial para la autonomía.

\title{
RESUMO
}

A formação inicial de professores implica não só preparar os estudantes para conhecer, pensar e compreender, mas também ajudá-los a compreender para atuar. Vários especialistas têm recomendado uma pedagogia de casos na formação inicial para a docência, uma vez que pode apoiar a superação de algumas carências dos modelos de formação tradicionais e contribuir de modo significativo para o desenvolvimento profissional dos professores. Uma pedagogia de casos baseia-se na ideia de que o conhecimento é construído com base no conhecimento prévio, está associado à experiência, é passível de transformação e pode mudar. Por outro lado, uma pedagogia de casos ajuda o professor em formação a compreender a complexidade da docência, encorajando a busca de soluções de ensino alternativas. Neste artigo, sustenta-se que uma pedagogia de casos é útil para promover a reflexão crítica, facilitar a compreensão da teoria e formar professores com capacidade de resolver problemas, favorecendo a relação entre o ensino, a investigação e o desenvolvimento profissional.

Palavras-chave: formação de professores; pedagogia de casos; formação inicial de professores para a autonomia.

\begin{abstract}
Initial teacher education does not only imply preparing student teachers for knowing, thinking and understanding but it involves supporting student teachers in developing understanding in order to act. Several specialists have recommended case pedagogy for initial teacher education, arguing that it can help teacher educators overcome some of the shortcomings of traditional models of teacher education, and it can greatly enhance teacher professional development. Case pedagogy is based on the idea that new knowledge is constructed in connection with prior knowledge, it is directly related to experience, it is transformable and it can change overtime. In addition, case pedagogy helps student teachers understand the complexity of teaching and this encourages experimentation and innovation in teaching. In this paper I maintain that case pedagogy encourages critical reflection, facilitates the
\end{abstract}


understanding of theory and it helps teachers to develop problem-solving skills, linking teaching, research and professional development.

Keywords: teacher education; case pedagogy; initial teacher education for autonomy.

\section{Introducción}

La formación inicial de profesores parece estar en permanente crisis por las dificultades inherentes a la propia tarea, pero sobre todo por la falta de apoyo institucional a la misma. Detrás de esta falta de apoyo subyace la creencia de que "cualquiera puede enseñar" y la duda de que la formación de profesores sea una disciplina digna de ser tomada en serio, lo que también refleja una actitud de indiferencia ante la educación de las nuevas generaciones. En el contexto español se sigue ignorando a nivel político que el factor más influyente en el aprendizaje es el profesor (HATTIE, 2003) y que es verdaderamente posible formar profesores excelentes. Rokoff (2004) descubrió grandes diferencias dependientes del profesor en los resultados de los alumnos, por lo que sugiere que la forma de mejorar el rendimiento de los alumnos pasa necesariamente por mejoras sustanciales en la formación del profesorado. En esta misma línea de razonamiento, Imbernón (2010) mantiene que el sistema educativo siempre ha situado la formación del profesor en el contexto de un discurso ambivalente, paradójico y, en ocasiones, simplemente contradictorio, pues por un lado nos encontramos con la retórica histórica de la relevancia de esta formación, y por otro la realidad, es decir, la escasa relevancia de la formación de los profesionales de la enseñanza en el sistema educativo.

En el contexto español, los resultados de las diferentes evaluaciones ponen de manifiesto unos resultados de la enseñanza no satisfactorios. Los malos resultados de las evaluaciones han generado preocupación en la sociedad española, ya que ponen en evidencia que el sistema educativo no está formando adecuadamente a los escolares. Abordar esta situación implicaría una reflexión sobre la calidad de la enseñanza primaria y secundaria en España. Esta reflexión debe incluir todos los aspectos de la problemática educativa, incluida la formación del profesorado, pues la solución a los problemas de la educación pasa por la persona que enseña, por el profesional que trabaja directamente con los alumnos en el aula, el profesional que interpreta la legislación educativa y se esfuerza por implementar el currículo oficial. Luego la solución está en la formación para la profesión docente, porque cuando los alumnos son enseñados por pro- 
fesionales con una buena formación pedagógica logran un mayor desarrollo de su potencial de aprendizaje.

En España la formación inicial que habilita para la docencia en la enseñanza secundaria desde el curso 2009-10 se realiza en un programa de máster de 60 créditos European Credit Transfer System (ECTS) (Cuadro 1), conducentes a conseguir la formación pedagógica y didáctica establecida en la Ley Orgánica 2/2006 de Educación (LOE) y en el Real Decreto 1834/2008 de 8 de noviembre.

\section{CUADRO 1 - ESTRUCTURA DEL MÁSTER UNIVERSITARIO EN FORMACIÓN DEL PROFESORADO DE EDUCACIÓN SECUNDARIA OBLIGATORIA Y BACHILLERATO, FORMACIÓN PROFESIONAL Y ENSEÑANZAS DE IDIOMAS}

\begin{tabular}{|c|c|c|}
\hline Módulo & Materia & Créditos \\
\hline Genérico & $\begin{array}{l}\text { - Aprendizaje y desarrollo de la personalidad } \\
\text { - Procesos y contextos educativos } \\
\text { - Sociedad, familia y educación }\end{array}$ & 12 \\
\hline Específico & $\begin{array}{l}\text { - Complementos de formación (6) } \\
\text { - Aprendizaje y enseñanza de las materias correspondientes (12) } \\
\text { - Innovación docente e iniciación a la investigación educativa (6) }\end{array}$ & 24 \\
\hline $\begin{array}{l}\text { Practicum } \\
\text { (Estágio) }\end{array}$ & $\begin{array}{l}\text { - Practicum en la especialización (10) } \\
\text { - Trabajo fin de máster (6) }\end{array}$ & 16 \\
\hline $\begin{array}{l}\text { Libre } \\
\text { disposición }\end{array}$ & $\begin{array}{l}\text { - Los alumnos pueden optar por cursar estos créditos en materias/ } \\
\text { asignaturas de otros másteres oficiales, en materias/asignaturas } \\
\text { de otras especialidades de este máster y/o en materias específicas } \\
\text { ofertadas por cada Universidad }\end{array}$ & 8 \\
\hline
\end{tabular}

FUENTE: El autor (2016).

Así, las enseñanzas del Máster de Profesorado de Secundaria, según él se ordenan en torno a los módulos de psicosociopedagogía, metodología didáctica y practicum, conocimientos que han sido considerados fundamentales para la adquisición de las competencias profesionales propuestas para los docentes, y que han de ofrecer la perspectiva necesaria para comprender y dotar de sentido la futura intervención educativa del profesorado de enseñanza secundaria.

Sin duda alguna, este máster ha supuesto una considerable mejora con respecto al modelo anterior de formación del profesorado de secundaria (Certificado de Aptitud Pedagógica - CAP), pues al programa se ha enmarcado dentro de los estudios de posgrado en el actual contexto universitario y la duración del mismo ha pasado de 150 a 600 horas. No obstante, siguen siendo muchas las deficiencias de este programa de formación, pero quizás la más serias son la puesta en práctica de un modelo de formación basado en competencias y la desconexión entre teoría y práctica educativas, pues no hay coordinación entre 
el profesorado de los diferentes módulos entre sí ni de estos con el practicum. La implantación del nuevo máster puede suponer un momento propicio para el cambio en el modelo de formación que los formadores ponemos en práctica. El modelo basado en competencias reclama un nuevo modelo de formación, que fomente el aprendizaje activo, que capacite al alumno para el uso de la información, pues la noción de competencia implica aplicación a la práctica profesional. El modelo de competencias reclama la integración de teoría y práctica, por lo que el acercamiento al mundo profesional es crucial. No basta entender lo que se aprende, sino que se trata de ser operativamente competente. (BARNETT, 2001). En la universidad española la adopción del modelo de competencias se ha hecho realidad a nivel de discurso político pero no tanto en el quehacer docente. Este modelo se ha adoptado sin una reflexión profunda sobre el alcance de este nuevo enfoque curricular a diferentes niveles. No obstante, sobre lo que no cabe duda es que un modelo que reivindica estar basado en el desarrollo de competencias profesionales no debería seguir el paradigma tecnológico basado en la transmisión de conocimiento experto, pues como aseguran Nunan y Lamb (1996) el enfoque tecnológico es deficiente, porque asume que la capacidad para enseñar se puede caracterizar en términos de conocimiento y destrezas preestablecidas, pero lo que hoy es tecnológicamente aceptado puede no serlo mañana. Fullan (2002) distingue entre problemas técnicos, que el conocimiento existente puede solucionar, y problemas de adaptación, que el conocimiento actual no puede solventar, y para los cuales son necesarias iniciativas innovadoras.

Aunque mi experiencia en la formación inicial se circunscribe a la especialidad de Inglés como lengua extranjera, en este trabajo me propongo analizar, describir y contextualizar la que considero como una estrategia innovadora y con un gran potencial para la formación inicial docente para la autonomía: la pedagogía de casos.

\section{Formación para la profesión docente}

Una de las implicaciones derivadas del Proceso de Bolonia es la necesidad de un nuevo paradigma de enseñanza superior centrada en el alumno, que fomente el aprendizaje a lo largo de la vida, que forme sujetos (profesores) capaces de utilizar el conocimiento, de actualizarse, de aprender permanentemente, de entender lo que aprenden, y todo ello de manera tal que sean capaces de adaptarlo a nuevas situaciones cambiantes y de fomentar esta capacidad en sus alumnos. Necesariamente, la sociedad del conocimiento tiene que ser también la 
sociedad del aprendizaje. Esta idea está intrínsecamente unida a una visión de la educación en un contexto más amplio: el aprendizaje a lo largo de la vida. Por su parte, el modelo de competencias adoptado exige también una renovación de los contenidos, métodos, prácticas y medios de transmisión del saber, que han de basarse en nuevos tipos de vínculos y de colaboración con la comunidad y con los más amplios sectores de la sociedad. El aprendizaje por competencias no consiste en la mera acumulación de conocimiento, sino que además implica comprender y usar pertinentemente dicho conocimiento.

En el último cuarto del s. XX, surgieron diversas propuestas post-positivistas englobadas bajo el paraguas del paradigma reflexivo, que enfatizan la necesidad de que el profesor se convierta en profesor reflexivo crítico y acentúan la centralidad de la experiencia en el desarrollo profesional docente. (SCHÖN, 1983, 1987; TABACHNICK; ZEICHNER, 1991; ZEICHNER, 1996). Este paradigma supera la propuesta de formación basada en la lógica que asume que se aprende a enseñar mediante el modelo academicista. En este cambio de paradigma, el aprendizaje centrado en el alumno puede jugar un papel importante por su énfasis en el aprendizaje, es decir, en el proceso más que en el producto, por ser una enseñanza que enfatiza la forma de aprender y los procesos que contribuyen al desarrollo del potencial de aprendizaje del alumno. En la sociedad del conocimiento, de la información y del aprendizaje, la mejora de los procesos y estrategias de razonamiento, el ayudar a aprender a pensar, el desarrollo de la autonomía del individuo, el aprender a aprender y el aprendizaje permanente constituyen los grandes retos de la educación. Para tal fin, los docentes nos vemos obligados a reinventar nuestra práctica para dar satisfacción a las demandas de la sociedad actual de docentes competentes en el ejercicio de la profesión, que a su vez, implementen en el aula aquellas estrategias metodológicas que mejor se adecuen a los objetivos propuestos en cada momento.

En este contexto es importante ser conscientes de los desafíos que el aprender a enseñar para la autonomía del alumno y del profesor supone. Así, el primero es que aprender a enseñar, en contra de lo que normalmente se piensa, no es una tarea fácil, sino una tarea compleja que implica, entre otras muchas cosas, aprender a enseñar de manera diferente a como fuimos enseñados, luego no se trata simplemente de aprender nuevo conocimiento, sino en gran parte de "desaprender" lo aprendido y de aprender nuevo conocimiento para ponerlo en práctica. (JIMÉNEZ RAYA, 2016). Para que un profesor pueda hacer frente con éxito a la complejidad que emana de la naturaleza cambiante de la enseñanza y del aprendizaje en el aula es importante que los profesores entiendan la naturaleza multidimensional de la clase. La enseñanza es una actividad extremadamente compleja, que tiene lugar en un complejo triángulo de relaciones entre el profesor, los alumnos y la materia objeto de estudio en el que los 
puntos del triángulo cambian constantemente. (McDONALD, 1992, p. 1). La formación inicial tiene, por tanto, que aspirar a que los profesores en formación conceptualicen el proceso didáctico como eminentemente dinámico, sometido a pluralidad de variaciones, dilemas y obstáculos, lo que plantea al profesor la necesidad de buscar constantemente alternativas de actuación. (VIEIRA, 2012). Y esta búsqueda constituye, de algún modo, un proceso de investigación cuyos resultados revertirán en el perfeccionamiento de la actividad didáctica cotidiana. A veces, como mantienen Munby, Russel y Martin (2001), la buena práctica docente refuerza la idea de que la enseñanza es una profesión que requiere poco esfuerzo, porque el conocimiento y la experiencia que la sustentan son invisibles a los alumnos. De ahí que los profesores noveles tiendan a imitar los aspectos superficiales de la enseñanza. Aunque observemos una buena práctica docente, es prácticamente imposible llegar a entender su complejidad. Esta complejidad requiere que los profesores se inspiren en varios tipos de conocimiento, contextos sociales, cultura escolar, currículo y enseñanza, integrando lo que saben para crear tareas motivadoras de aprendizaje, por lo que separar teoría y práctica en la formación da origen a una falsa dicotomía. La formación inicial tampoco puede basarse exclusivamente en el cómo, excluyendo el porqué. Aprender a enseñar requiere aprender a pensar y a actuar como un profesor. (KENNEDY, 1999).

Hay que avanzar en la formación del profesorado desde el presupuesto de que el conocimiento profesional es acumulativo, se va construyendo desde estructuras de conocimiento previas y tiene un importante componente biográfico y narrativo. Además debemos avanzar desde la humildad que significa reconocer que difícilmente un nuevo planteamiento curricular vaya a ser la solución definitiva a los complejos problemas de la enseñanza de lenguas extranjeras. Nuestro convencimiento es que toda opción metodológica debería ser vista como un paso más hacia la mejora, pero reconociendo que carece de carácter definitivo y que con toda seguridad presentará lagunas irresolubles. Otro gran reto al que nos enfrentamos es la necesidad de ayudar desde la formación inicial a superar la desmotivación existente entre el profesorado en el contexto español. Para tal fin, se hace preciso ir sustituyendo las políticas y las prácticas formativas centradas en el modelo academicista por políticas y prácticas activas basadas en la experiencia (VIEIRA, 2009), que integren teoría y práctica de forma contextualizada, que, además, desde el primer momento ayuden al alumno en formación a pensar como un profesor, presenten la profesión docente como una constante búsqueda de la perspectiva del alumno y motiven para la formación permanente, poniendo énfasis en la autonomía del alumno y del profesor, pues la relación entre autonomía y motivación ha quedado suficientemente demostrada en múltiples estudios realizados en el marco de la teoría de la Autodeterminación en contextos muy diversos. 
La formación inicial necesita dotarse de un sistema de orientación concebido como un proceso continuo de ayuda al docente en formación, que le permita conocer sus necesidades formativas, motivarlo para la formación presente y para el ejercicio de la profesión en su momento, fomentando actitudes favorables hacia la incorporación de nuevas competencias profesionales que respondan a los grandes retos de la educación en el siglo XXI. Preparar a alguien para la profesión docente no solamente implica prepararlo para conocer, pensar y entender lo que necesita entender para practicar, esto sería solamente el principio, sino que la preparación para la profesión docente implica enseñar a alguien a entender para actuar, a actuar para que en el ejercicio de su profesión pueda dejar una huella indeleble en las mentes y vidas de otros. Preparar para la profesión docente significa preparar para conocer, actuar y practicar, para tal fin es necesario suscribir y poner en práctica un programa "fuerte" de desarrollo profesional del profesorado (JOYCE; SHOWERS, 1988), que esté diseñado para enfrentar la tendencia natural de todo profesor a enseñar como fue enseñado si queremos evitar que el sistema de enseñanza se perpetúe. Se necesita, pues, un programa que les ayude a construir de forma consciente su identidad profesional, su visión ideal de la educación en línea con el estado de la investigación sobre los procesos de enseñanza y de aprendizaje. (JIMÉNEZ RAYA, 2016).

Nuestro postulado es que un modelo fuerte de desarrollo profesional tiene que incorporar una visión ilusionante y motivadora de la educación (KINCHELOE, 2003, p. 111) y tener en cuenta los postulados básicos de la teoría y práctica de la formación de profesores. En mi caso, la visión ilusionante de la educación es la pedagogía para la autonomía (JIMÉNEZ RAYA; LAMB; VIEIRA, 2007), pues el objetivo último de la educación es la autonomía del individuo. El modelo "fuerte" de formación para la profesión docente lo encarna la pedagogía de casos. (JIMÉNEZ RAYA; VIEIRA, 2015).

\section{La pedagogía de casos en la formación inicial del profesor}

Diversos especialistas (CARTER; RICHARDSON, 1989; JIMÉNEZ RAYA; VIEIRA, 2015; J. SHULMAN, 1987, 1996, 2004) han recomendado la pedagogía de casos para la formación inicial, argumentando que puede ayudar a superar algunas de las carencias de los modelos tradicionales de formación y contribuir de manera muy significativa al desarrollo profesional de los profesores, pues los casos nos pueden dar una visión o imagen de lo que es posible en el aula. De forma específica ha sido recomendada para la formación inicial 
como medio para superar algunas de las carencias de las experiencias prácticas, ya que facilita el desarrollo profesional. (CARTER; RICHARDSON, 1989; J. SHULMAN, 1987, 1992). La pedagogía de casos también se utiliza para generar teoría a partir de la práctica y para aplicar la teoría a la práctica. (HARRINGTON, 1995). La relación entre estos dos tipos de conocimiento es un aspecto crucial en el aprendizaje para la profesión docente. (KESSELS; KORTHAGEN, 1996).

La pedagogía de casos se fundamenta en la idea de que el conocimiento se construye sobre la base del conocimiento previo, está asociado a la experiencia, es situado y producto de la actividad, del contexto y de la cultura en la que se desarrolla y se utiliza, es además transformable y puede cambiar. De ahí la reivindicación de que los casos ayudan al profesor en formación a entender la complejidad de la docencia y del aprendizaje, animando a la búsqueda de soluciones docentes alternativas además de obligarle a pensar como profesor. En general, el objetivo del uso de la pedagogía de casos en la formación para la profesión docente es presentar de forma integrada el conocimiento, las destrezas y las actitudes que subyacen a la enseñanza para crear las condiciones que permitan a los profesores entender la complejidad de la docencia.

El uso genérico del término "caso" hace referencia a una situación, suceso, acontecimiento, conjunto de circunstancias, etc., entendidos como una entidad particular y diferenciada. En la bibliografía especializada se mantiene que un caso es una representación del desarrollo de la comprensión humana enraizada en historias de logros humanos en un determinado contexto histórico cultural. Grossman (1992) se pregunta si los casos son ejemplos de teoría que ejemplifican el aprendizaje teórico o, si por el contrario, son ejemplos de la práctica de los que los alumnos pueden aprender sobre la enseñanza. Para L. Shulman (1992, p. 3), un caso es una descripción de un episodio práctico, una selección de la realidad, una rodaja de la vida, una historia diseñada y presentada como material de estudio, un ejercicio, un puzle o un problema. Para gran parte de los investigadores, un caso es una narrativa o descripción de un episodio de enseñanza que ha sido elaborado para su uso en la formación de profesores. Los casos normalmente incluyen los puntos de vista y las creencias del profesor que protagoniza el caso y/o las del escritor del caso. Asimismo, de un caso se espera que sea rico en detalles sobre los dilemas y desafíos descritos. (SHULMAN; SATO, 2006). Los casos también pueden incluir reflexiones del profesor o del autor sobre el tema del caso. El objetivo último es suscitar preguntas sobre los aspectos más relevantes de la enseñanza y del aprendizaje, ya que en caso contrario el caso tiene poco valor. Se espera además que los casos sean representativos de los dilemas, problemas y obstáculos a los que se enfrentan los profesores a diario en el aula, que no sean simplemente una historia, sino que, además de la descripción, presenten de forma coherente una abundancia de datos. Igualmente, se espera 
que los casos sean un caso sobre algo (SHULMAN, 1986, p. 11), que ilustre, que ejemplifique, pero que, además, incorpore principios teóricos, pues como asegura Shulman (1986), son las herramientas más poderosas que puede tener un profesor. Los casos que reúnen estas características y describen episodios o situaciones complejas generan debates profundos sobre diferentes aspectos de la práctica profesional. (RICHERT, 1991). Desde mi perspectiva, un caso es un intento de capturar mediante la narración y la descripción la práctica docente de un profesor para mostrarla en toda su complejidad a otros profesores con el objetivo último de ayudarles a entender el porqué y el cómo de la pedagogía para la autonomía.

Múltiples autores han escrito sobre las razones que avalan el uso de la pedagogía de casos en la formación de profesores. L. Shulman $(1986,1992)$, por ejemplo, sugiere que los casos se pueden utilizar para enseñar principios y conceptos de naturaleza teórica, antecedentes prácticos, cuestiones de moral y ética, estrategias, inclinaciones y tendencias así como hábitos mentales. La psicología cognitiva juega aquí un papel clave, pues nos ayuda a entender este enorme potencial de la pedagogía de casos. Así, el trabajo de Spiro y sus colegas sobre el aprendizaje en ámbitos mal estructurados (SPIRO et al., 1988), entre los que se encuentra la enseñanza, sugiere que la pedagogía de casos puede ayudar a los profesores a hacer frente a la complejidad del aprendizaje y la enseñanza. Además, puesto que los casos por su propia naturaleza son narraciones contextualizadas y situadas, podemos mantener que la pedagogía de casos también tiene en cuenta los postulados de la psicología cognitiva sobre la naturaleza "situada" del conocimiento y del pensamiento. (BROWN; COLLINS; DUGUID, 1989).

Otra característica relevante de los casos es su enorme potencial para producir el cambio de las creencias de los profesores en formación. El papel de las creencias en la formación inicial y continua ha sido ampliamente estudiado (CHENG; CHENG; TANG, 2010; PAJARES, 1992; WILSON; COONEY, 2002) con la conclusión de que las creencias formadas tempranamente y durante un período prolongado de tiempo son intrínsecamente resistentes al cambio. Asimismo se especula con la idea de que jueguen un papel primordial en el aprendizaje, pues la investigación apunta a que éstas filtran la información que los profesores en formación docente reciben (HOLT-REYNOLDS, 1992; JOHNSON, 1994; KAGAN, 1992; PAJARES, 1992) por lo que la formación inicial no puede limitarse a presentar al alumno con nuevas ideas, sino que ha de hacerlo de forma que se facilite el cambio conceptual en lugar de limitarse simplemente a la trasmisión de conocimientos y al fomento de la imitación de determinadas prácticas. Al hilo de esta reflexión, la teoría del cambio conceptual (POSNER et al., 1982) mantiene que la modificación de creencias profundamente arraigadas requiere la confrontación con información e imágenes discordantes 
para provocar la disonancia. De acuerdo con este modelo, aunque puedan existir más condiciones para el cambio conceptual, hay cuatro de especial relevancia (POSNER et al., 1982, p. 214): (1) el individuo debe sentirse insatisfecho con las concepciones existentes. Es improbable que se produzca el cambio en el sistema de creencias, sino se percibe la discrepancia entre las creencias actuales y la alternativa presentada; (2) el modelo alternativo debe ser inteligible para que permita la exploración de posibilidades; (3) la alternativa que se sugiere debe ser factible y vívida para que cause impresión. Es altamente aconsejable que la presentación del modelo alternativo vaya acompañado de experiencias y preguntas que obliguen al profesor en formación a identificar posibles discrepancias entre los paradigmas existente y nuevo; (4) el nuevo modelo debe tener un mayor potencial de mejora del aprendizaje y de las relaciones profesor alumno, y ser aplicable en diferentes contextos.

La formación inicial (para la autonomía del alumno y del profesor) tiene que centrarse en el desarrollo de actitudes positivas que predispongan para la aceptación de nuevos modelos docentes y para la adquisición de las competencias profesionales que capaciten para el aprendizaje autónomo. Lamentablemente, esto por sí solo no es suficiente. Es necesario que los programas de formación docente creen las condiciones para que los profesores en formación aumenten su conocimiento y sus destrezas, fomenten su desarrollo profesional. En mi caso, me propongo además como objetivo ayudarles a adquirir conocimiento práctico directamente relacionado con el quehacer docente del día a día del profesor, pues los programas que no tienen en cuenta estas premisas básicas están condenados al fracaso. Otro aspecto crucial que debe tener en cuenta un programa de formación inicial es el empoderamiento de los profesores para que se conviertan en protagonistas de su vida profesional, luego la autonomía, la responsabilidad, la reflexión y la indagación son necesariamente los objetivos últimos de la formación para la profesión docente. Como asegura L. Shulman (2004, p. 514), el aprendizaje del profesor es más activo cuando incorpora la experimentación y la indagación, también cuando incorpora la escritura, el diálogo y se plantean interrogantes sobre la práctica y la teoría. Los enfoques del aprendizaje activo se apoyan en la creencia de que se facilita el aprendizaje cuando logramos incorporar en las actividades de formación el conocimiento y el significado mediante la exploración, la experimentación y a manipulación de ideas. (MEYERS; JONES, 1993). En la pedagogía de casos teoría y práctica están inextricablemente unidas, por lo que la dicotomía sobre el papel de ambas queda resuelta: las dos son necesarias.

Jiménez Raya y Vieira (2015) describen dos tipologías de casos que se diferencian por el autor y los usos pedagógicos que de ellos se pueden hacer en la formación de profesores. El primero se refiere a los casos escritos por exper- 
tos o por profesores utilizados como precedentes y ejemplos para la reflexión, el análisis y la interpretación, aunque también pueden ser también utilizados como ejemplos para ilustrar la teoría. El segundo uso se refiere al desarrollo, experimentación de alternativas pedagógicas y escritura de un caso por parte del profesor en formación. Los dos usos de pedagogía de casos son necesarios en la formación para la profesión docente, pues enfatizan aspectos y destrezas diferentes en el profesor.

Sin duda, ambos son de gran valor formativo, pero con diferente énfasis. Los primeros se utilizan como ejemplos contextualizados de principios y prácticas docentes con el objetivo de fomentar el pensamiento crítico y la reflexión del profesor sobre la base de un caso real. El segundo uso, pone al profesor en la necesidad de identificar un aspecto mejorable de su práctica o una nueva metodología o estrategia docente que le gustaría experimentar en el aula, lo cual exige que se documente a nivel teórico y práctico, planifique una experiencia de intervención a pequeña escala, la ponga en práctica, tome notas, recoja información y que elabore una memoria (un caso) sobre la experiencia, por lo que el profesor que elabora un caso se está entrenando en la indagación sistemática e informada y en la reflexión crítica. A su vez, estos casos escritos por los propios profesores en formación pueden pasar a engrosar el banco de casos disponibles para su utilización como herramientas de discusión sobre la enseñanza en el futuro. La escritura de casos hace de la formación una experiencia verdaderamente enriquecedora y transformadora, combinando teoría y práctica de forma natural, fomentando actitudes positivas hacia la innovación, hacia la reinvención constante de la práctica docente. Además son el puente natural a la integración del módulo teórico con el practicum. Jiménez Raya y Vieira (2015) describen la siguiente secuencia pedagógica de la escritura de casos por parte de los profesores en formación: (1) Análisis de casos escritos por otros profesores en años previos; (2) Construcción del caso, incluyendo el diseño, experimentación, análisis y escritura del caso.

Las posibilidades de incorporación de la pedagogía de casos en la formación inicial son muchas, pero, creo que el gran potencial está en la posibilidad que ofrece para unir y coordinar la formación entre los módulos "teóricos" y el practicum. Así, en mi práctica como formador utilizo los casos como precedentes y prototipos para la discusión y el debate en los módulos "teóricos" de la formación inicial, mientras que la escritura de casos la utilizo para establecer nexos entre teoría y práctica y para fomentar la colaboración entre módulos, la experimentación, la innovación y la indagación. El desarrollo del caso tiene lugar durante la fase de prácticas en el centro educativo. Mi papel consiste en ayudar a los alumnos a identificar un aspecto relacionado con la pedagogía para la autonomía que desean experimentar en el aula, orientar en la planificación y 
en la escritura del caso, fomentando en la medida de lo posible la implicación de los profesores tutores. La elaboración de una narrativa sobre el caso favorece la conexión entre conocimiento formal y conocimiento práctico, estimulando la reflexión y la adquisición de los diferentes conceptos. La escritura de un caso pone el énfasis en la innovación, la experimentación, la reflexión, la indagación y la exploración del espacio de lo posible en la docencia. Es más, se puede y debería fomentar que el trabajo fin de máster fuese la construcción de un caso, pues permitiría además de los beneficios citados con anterioridad hacer una evaluación global del grado de adquisición de las competencias profesionales de los profesores en formación. El profesor que escribe su caso se convierte en investigador, pues se ve obligado a analizar su propia práctica críticamente, convirtiéndose además en generador de nuevo conocimiento sobre la docencia. Aprender a construir un caso requiere un proceso complejo de indagación sobre la propia práctica, la investigación sobre experiencias previas relacionadas, la reflexión profunda, la organización de las ideas, la verificación del conocimiento y su validación.

\section{Conclusión}

En este trabajo he argumentado sobre la base de mi experiencia profesional a favor de la pedagogía de casos y mantenido que la pedagogía de casos es útil para promover la reflexión crítica, facilitar la comprensión de la teoría y para formar profesores con capacidad de resolución de problemas y pensamiento crítico, ya que favorece la conexión entre enseñanza, investigación y desarrollo profesional.

La pedagogía de casos nos permite evitar que nuestra práctica formativa se limite exclusivamente a proporcionar a los futuros docentes las piezas de un puzle que no conocen, y que irremediablemente acaban por no encajar entre sí, si es que el futuro docente llega en algún momento a plantearse la necesidad de intentar hacerlas encajar. Dado que cada formador concibe la enseñanza de modo diferente y tendemos a trasmitir los contenidos de nuestras materias como un fin en sí mismo, como si se justificaran por el solo hecho de formar parte del currículo, lo que en realidad estamos haciendo es dificultar que los futuros profesores comprendan el objetivo y la utilidad de estos saberes así como su relación con la práctica docente, por lo que perpetuamos la división entre teoría y práctica docente. La formación docente tiene que acabar con la práctica que reduce el currículo a la simple provisión de saberes sin conexión 
entre sí. Si miramos hacía nuestra propia práctica docente, es posible que nos demos cuenta de que nuestras creencias y nuestra práctica docente no concuerdan. Las consecuencias son la perpetuación de prácticas docentes centradas en el profesor y en el libro de texto.

Las características propias de nuestra profesión determinan que en nuestro caso investigación y docencia confluyan estrechamente en el mismo ámbito de actuación y que nuestros proyectos y resultados de trabajo se influyan de manera relevante. Teoría y práctica están, pues, tan estrechamente vinculadas, que no se debería dar una dicotomía entre estructuras de investigación y estructuras de enseñanza.

Los momentos de cambio como el que todavía está teniendo lugar en la universidad española con el Proceso de Bolonia pueden ser el momento para el cambio de paradigma en la formación inicial. Los formadores debemos reinventar nuestra práctica, buscando modelos "fuertes" de formación, experimentando nuevas metodologías que se adecuen a las nuevas necesidades educativas, pues los profesores que ahora formamos en el futuro se enfrentarán a cambios difíciles de imaginar en estos momentos. De ahí la necesidad de estrategias que inciten al análisis de los problemas pedagógicos, a reflexionar sobre las alternativas posibles y a desarrollar el hábito de la investigación/indagación informada sobre la propia práctica docente.

\section{REFERENCIAS}

BARNETT, R. Los límites de la competencia. El conocimiento, la educación superior y la sociedad. Barcelona: Gedisa, 2001.

BROWN, J. S.; COLLINS, A.; DUGUID, P. Situated cognition and the culture of learning. Educational Researcher, v. 18, n. 1, p. 32-42, 1989.

BRUNER, J. Realidad mental y mundos posibles. Barcelona: Gedisa, 1980.

CARTER, K.; RICHARDSON, V. A curriculum for an initial-year-of-teaching program. Elementary School Journal, v. 89, n. 4, p. 405-419, 1989.

CHENG, M.; CHENG, A.; TANG, S. Closing the gap between the theory and practice of teaching: implications for teacher education programs in Hong Kong. Journal of Education for Teaching: International Research and Pedagogies, v. 36, n. 1, p. 91-104, 2010.

CHRISTENSEN, C. R.; GARVIN, D. A.; SWEET, A. Education for judgment: the artistry of discussion leadership. Boston: Harvard Business School Press, 1991. 
FULLAN, M. Los nuevos significados del cambio en la educación. Barcelona: Octaedro, 2002.

GROSSMAN, P. L. Teaching and learning with cases. In: SHULMAN, J. (Org.). Case methods in teacher education. New York: Teachers College Press, 1992. p. 227-239.

HARRINGTON, H. L. Fostering reasoned decisions: case-based pedagogy and the professional development of teachers. Teaching and Teacher Education, v. 11, n. 3, p. 203-214, 1995.

HATTIE, J. Teachers make a difference: what is the research evidence? Plenaria impartida en el Congreso anual: Building Teacher Quality de la Australian Council for Educational Research, 2003. Disponible en: <http:/www.educationalleaders.govt.nz/ Pedagogy-and-assessment/Building-effective-learning-environments/Teachers-Makea-Difference-What-is-the-Research-Evidence>. Acceso en: 30 ago. 2016.

HOLT-REYNOLDS, D. Personal history-based beliefs as relevant prior knowledge in course work. American Educational Research Journal, v. 29, n. 2, p. 325-349, 1992.

IMBERNÓN, F. La profesión docente en el nuevo contexto educativo. In: CARNICERO DUQUE, P.; SILVA GARCÍA, P.; MENTADO LABAO, T. (Org.). Nuevos retos de la profesión docente. II Seminario Internacional RELFIDO. Barcelona: Collecció FODIP, 2010. p. 6-7.

JIMÉNEZ RAYA, M. Innovación en la formación inicial para la autonomía: el potencial del yo posible en la construcción de la identidad profesional. In: VIEIRA, F.; COELHO DA SILVA, J. L.; FLORES, M. A.; COSTA OLIVEIRA, C.; FERREIRA, F. I.; CAIRES, S.; SARMENTO, T. (Org.). Innovação pedagógica no Ensino Superior: ideias (e) práticas, v. 2. Santo Tirso: De Facto Editores, 2016. p. 151-163.

JIMÉNEZ RAYA, M.; LAMB, T.; VIEIRA, F. Pedagogy for autonomy in language education in Europe - towards a framework for learner and teacher development. Dublin: Authentik, 2007.

JIMÉNEZ RAYA, M.; VIEIRA, F. Enhancing autonomy in language education: a casebased approach to teacher and learner development. New York: Mouton de Gruyter, 2015.

JOHNSON, K. E. The emerging beliefs and instructional practices of preservice English as a second language teachers. Teaching and Teacher Education, v. 10, n. 4, 1994, p. 439-452.

JOYCE, B.; SHOWERS, B. Staff development and student achievement. New York: Longman, 1988.

KAGAN, D. M. Implication of Research on Teacher Belief. Educational Psychologist, v. 27, n. 1, p. 65-90, 1992.

KENNEDY, M. The role of preservice teacher education. In: DARLING-HAMMOND, L.; SYKES, G. (Org.). Teaching as the learning profession: handbook of policy and practice. San Francisco: Jossey-Bass, 1999. p. 54-85. 
KESSELS, J.; KORTHAGEN, F. The relationship between theory and practice: back to the classics. Educational Researcher, v. 25, n. 3, p. 17-23, 1996.

KINCHELOE, J. Teachers as researchers - qualitative inquiry as a path to empowerment. London: Routledge Falmer, 2003.

McDONALD, J. P. Teaching: making sense of an uncertain craft. New York: Teachers College Press, 1992.

MEYERS, C.; JONES, T. B. Promoting active learning: strategies for the College Classroom. San Francisco: Jossey-Bass, 1993.

MUNBY, H.; RUSSELL, T.; MARTIN, A. K. Teachers' knowledge and how it develops. In: RICHARDSON, V. (Org.). Handbook of research on teaching. Washington, DC: AERA, 2001. p. 887-904.

NUNAN, D.; LAMB, C. The self-directed teacher: managing the learning process. Cambridge: Cambridge University Press, 1996.

PAJARES, M. F. Teachers' beliefs and educational research: cleaning up a messy construct. Review of Educational Research, v. 62, n. 3, p. 307-332, 1992.

POSNER, G. J.; STRIKE, K. A.; HEWSON, P. W.; GERTZOG, W. A. Accommodation of a scientific conception: toward a theory of conceptual change. Science Education, v. 66, p. 211-227, 1982.

RICHERT, A. E. Case methods and teacher education: using cases to teach teacher reflection. In: TABACHNICK, R. B.; ZEICHNER, K. M. (Org.). Issues and practices in inquiry-oriented teacher education. London: Falmer Press, 1991. p. 130-150.

ROCKOFF, J. E. The impact of individual teachers on student achievement: Evidence from panel data. The American Economic Review, v. 94, n. 2, p. 247-252, 2004.

SCHÖN, D. A. The reflective practitioner: how professionals think in action. New York, NY: Basic Books, 1983.

SCHÖN, D. A. Educating the reflective practitioner: toward a new design for teaching and learning in the professions. San Francisco, CA: Jossey-Bass, 1987.

SHULMAN, J. H. From veteran parent to novice teacher: a case study of a student teacher. Teaching and Teacher Education: An International Journal of Research and Studies, v. 3, n. 1, p. 13-27, 1987.

SHULMAN, J. H. Happy accidents: cases as opportunities for teacher learning. Paper presented at the annual meeting of the American Educational Research Association, New Orleans, LA, 2002. Disponible en: <http://cet.usc.edu/resources/teaching_learning/docs/ happy_accidents.pdf $>$.Acceso en: 15 sep. 2012.

SHULMAN, J. H.; SATO, M. Mentoring teachers toward excellence: supporting and developing highly qualified teachers. San Francisco: Jossey-Bass Publishers, 2006. 
SHULMAN, L. Those who understand: knowledge growth in teaching. Educational Researcher, v. 15, n. 2, p. 4-14, 1986.

SHULMAN, L. Knowledge and teaching: fof a new reform. Harvard Educational Review, v. 57, n. 1, p. 1-22, 1987.

SHULMAN, L. Toward a pedagogy of cases. In: SHULMAN, J. H. (Org.). Case methods in teacher education. New York: Teachers College Press, 1992. p. 1-30.

SHULMAN, L. Just in case: reflections on learning from experience. In: COLBERT J.; DESBERG, P.; TRIMBLE, K. The case for education: contemporary approaches for using case methods. Boston: Allyn \& Bacon, 1996. p. 197-217.

SHULMAN, L. The wisdom of practice - essays on teaching, learning, and learning to teach. San Francisco: Jossey Bass, 2004.

SPIRO, R. J.; COULSON, R. L.; FELTOVICH, P. J.; ANDERSON, D. K. Cognitive flexibility theory: advanced knowledge acquisition in ill-structured domains. In: PATEL, V. (Org.). Proceedings of the 10th Annual Conference of the Cognitive Science Society. Hillsdale, NJ: Erlbaum, 1988. p. 377-383.

TABACHNICK, B. R.; ZEICHNER, K. Issues and practices in inquiry-oriented teacher education. London: Falmer Press, 1991.

VIEIRA, F. Para uma pedagogia da experiência na formação pós-graduada de professores. Indagatio Didactica, v. 1, n. 1, p. 32-75, 2009.

VIEIRA, F. O lugar da experiência na formação de professores. In: SOARES, S. R.; BORBA, V. C. (Org.). Ensino e aprendizagens: análise de práticas. Salvador: EDUNEB, 2012. p. 25-75.

WILSON, M.; COONEY, T. Mathematics teacher change and development. In: LEDER, G. C.; PEHKONEN, E.; TORNER, G. Beliefs: ahidden variable in mathematics education? New York: Springer, 2002. p. 127-147.

ZEICHNER, K. Teachers as reflective practitioners and the democratization of schools reform. In: ZEICHNER, K.; MELNICK, S.; GOMEZ, M. L. (Org.). Currents of reform in preservice teacher education. New York: Teachers College Press, 1996. p. 199-214.

ZEICHNER, K.; LISTON, D. Teaching student teachers to reflect. Harvard Educational Review, v. 57, p. 23-48, 1987.

Texto recibido en 03 de octubre de 2016. Texto aprobado en 04 de noviembre de 2016. 
